\title{
Effects of Reagent Rotation on Stereodynamics Information of the Reaction $\mathrm{O}\left({ }^{1} \mathrm{D}\right)+\mathrm{H}_{2}(\mathrm{v}=\mathbf{0}, \mathrm{j}=\mathbf{0 - 5}) \rightarrow \mathrm{OH}+\mathrm{H}$ : A Theoretical Study
}

\author{
Da Kuang, Tianyun Chen, Weiping Zhang, ${ }^{*}$ Ningjiu Zhao, ${ }^{\dagger}$ and Dongjun Wang ${ }^{\dagger, *}$ \\ School of Materials Science and Engineering, Dalian University of Technology, Dalian 116024, China \\ ${ }^{*}$ E-mail: zhangwpdlut@126.com \\ 'Department of Chemistry, Hebei Normal University of Sciences and Technology, Qinhuangdao 066600, China \\ Received May 10, 2010, Accepted August 19, 2010
}

\begin{abstract}
Quasiclassical trajectory (QCT) method has been used to investigate stereodynamics information of the reaction $\mathrm{O}\left({ }^{1} \mathrm{D}\right)+\mathrm{H}_{2} \rightarrow \mathrm{OH}+\mathrm{H}$ on the DK (Dobbyn and Knowles) potential energy surface (PES) at a collision energy of $23.06 \mathrm{kcal} /$ mol, with the initial quantum state of reactant $\mathrm{H}_{2}$ being set for $\mathbf{v}=0$ (vibration quantum number) and $\mathbf{j}=0-5$ (rotation quantum number). The PDDCSs (polarization dependent differential cross sections) and the distributions of $P\left(\theta_{r}\right), P\left(\theta_{r}\right)$, $\mathrm{P}\left(\theta_{r}, \varnothing_{r}\right)$ have been presented in this work. The results demonstrate that the products are both forward and backward scattered. As $\mathbf{j}$ increases, the backward scattering becomes weaker while the forward scattering becomes slightly stronger. The distribution of $P\left(\theta_{r}\right)$ indicates that the product rotational angular momentum $\mathbf{j}$ ' tends to align along the direction perpendicular to the reagent relative velocity vector $\mathbf{k}$, but this kind of product alignment is found to be rather insensitive to $\mathbf{j}$. Furthermore, the distribution of $P\left(\sigma_{r}\right)$ indicates that the rotational angular momentum vector of the $\mathrm{OH}$ product is preferentially oriented along the positive direction of $y$-axis, and such product orientation becomes stronger with increasing $\mathbf{j}$.
\end{abstract}

Key Words: Vector correlation, $\mathrm{O}\left({ }^{1} \mathrm{D}\right)+\mathrm{H}_{2} \rightarrow \mathrm{OH}+\mathrm{H}$ reaction, Quasiclassical trajectory (QCT) method, Polarization dependent differential cross section(PDDCS)

\section{Introduction}

The elementary reaction $\mathrm{O}\left({ }^{1} \mathrm{D}\right)+\mathrm{H}_{2} \rightarrow \mathrm{OH}+\mathrm{H}$ has been extensively studied from both theoretical and experimental sides due to its fundamental role in atmospheric and combustion chemistry. Specifically, this reaction is also a prototype for insertion reaction in which the barrierless pathway leads to the formation of a $\mathrm{H}_{2} \mathrm{O}$ complex in the deep molecular well dominating the potential energy surface (PES) as demonstrated with different theoretical methods as well as experimental techniques. ${ }^{1-22}$

On the experimental side, Koppe and co-workers ${ }^{20}$ determined the absolute rate constant $\mathbf{k}=(2.7 \pm 0.6) \times 10^{-10} \mathrm{~cm}^{3} \mathrm{~s}^{-1}$ molecule $^{-1}$ and absolute reactive cross section $\sigma_{R}=7.6 \pm 1.5 \AA^{2}$ at the collision energy of $0.12 \mathrm{eV}$ for the reaction $\mathrm{O}\left({ }^{1} \mathrm{D}\right)+\mathrm{H}_{2} \rightarrow$ $\mathrm{OH}+\mathrm{H}$. Very recently, the non-adiabatic dynamics for $\mathrm{O}\left({ }^{1} \mathrm{D}\right)+$ $\mathrm{H}_{2}, \mathrm{D}_{2}$, and $\mathrm{HD}$ as well as for other systems have been studied with QM scattering methods ${ }^{4,5}$ and quasiclassical trajectory surface hopping (TSH) method, ${ }^{3}$ of which Han and co-work$\mathrm{ers}^{18,21}$ firstly introduced the benchmark non-adiabatic time-dependent quantum wave packet approach to study non-adiabatic dynamics of chemical reaction. On the theoretical side, Song and Gislason ${ }^{23}$ studied the effect of reagent vibration and rotation on the reaction of $\mathrm{O}+\mathrm{H}_{2}(\mathbf{v}=0,1,2 ; \mathbf{j})$ using quasiclassical calculations on Johnson-Winter potential energy surface. ${ }^{24}$ They found that for the reaction $\mathrm{O}+\mathrm{H}_{2}(\mathbf{v}=0, \mathbf{j})$ the reactive cross section $\mathbf{Q}_{\mathbf{R}}$ increases monotonically with rotation quantum number j. But for $v=1$ and 2, $\mathbf{Q}_{\mathbf{R}}$ shows quite different behaviors with respect to $\mathbf{j}$, i.e., near the threshold $\mathbf{Q}_{\mathbf{R}}$ rises from $\mathbf{j}=0$ to 2, then declines to a minimum at $\mathbf{j}=5$, and finally rises monotonically to $\mathbf{j}=10$. Alexander and co-workers ${ }^{8}$ calculated the differential cross sections for the reaction $\mathrm{O}\left({ }^{1} \mathrm{D}\right)+\mathrm{HD} \rightarrow \mathrm{OH}+\mathrm{D}$ with the me- thod of quasi-classical trajectory (QCT) at a collision energy of $0.197 \mathrm{eV}(19 \mathrm{~kJ} / \mathrm{mol})$ by using the potential energy surface of Schinke and Lester. ${ }^{2}$ They discovered a strong dependence of the differential scattering cross section on the product quantum state.

However, most of the previous experimental and theoretical studies for this chemical reaction are focused on its aspect of scalar properties such as integral reaction cross section, differential reaction cross section, rate constant, reaction probability and product rovibrational distributions, etc. In order to understand the title reaction more comprehensively, it is important to study not only its scalar properties, but also vector properties. Only by understanding together the above properties can the fullest picture of the scattering dynamics be obtained. The vectors such as $\mathbf{k}, \mathbf{k}^{\prime}$ (the reagent and products relative velocity vectors) and $\mathbf{j}^{\prime}$ (the product rotational angular momentum) can give complete depiction of the scattering dynamics. Angular distribution describing relative orientation of these vectors in space may be termed as the $\mathbf{k}, \mathbf{k}^{\prime}$, and $\mathbf{j}^{\prime}$ distribution. Vector properties, such as velocities and angular momentum, possess not only magnitude that can be directly related to transnational and rotational energies, but also well-defined directions. Undoubtedly, one of the most important things is determining the product rotational alignment and orientation about the reagent relative velocity vector. ${ }^{25}$ The dynamical stereochemistry mainly investigates vector properties, such as the angle between reagent and product, product scattering angle distribution, correlation between product angular momentum and relative velocity vectors. With some pioneering studies undertaken by Fano, Herschbach, and co-workers, ${ }^{26,27}$ the dynamical stereochemistry is becoming an exceedingly attractive field in che- 
mical physics in recent decades. Han and his team ${ }^{25,28-32}$ have done lots of excellent work in this area and obtained some important results. They developed a quasiclassical trajectory (QCT) method to calculate the trajectory of light light-light, heavy heavy-light, heavy light-light, and light heavy-light mass combination reactions on attractive and repulsive potential energy surfaces. With this method, they investigated the influence of reaction location of energy barrier on the product alignment of reaction $\mathrm{A}+\mathrm{BC}$ and the dependence relation of product rotational alignment on different collision energies. Recently, significant theoretical and experimental processes about the vector properties of the $\mathrm{O}\left({ }^{1} \mathrm{D}\right)+\mathrm{H}_{2}$ reaction have been made by some other researchers. Casavecchia and co-workers ${ }^{11}$ used mass spectrometric detection of the $\mathrm{OH}$ molecule for the measurement of angular and velocity distributions of the products for the $\mathrm{O}\left({ }^{1} \mathrm{D}\right)+\mathrm{H}_{2}$ reaction at $\mathrm{E}_{\mathrm{col}}=0.08,0.13$ and $0.4 \mathrm{eV}$. For the lower collision energy, they discovered the angular distribution is backward symmetric and for the higher one $\left(\mathrm{E}_{\mathrm{col}}>0.1 \mathrm{eV}\right)$ a slight asymmetry favoring backward peaking appears. The measurement of product polarization as a function of scattering angle has been achieved with the development of photoloc method. ${ }^{33-37}$ Most recently, $\mathrm{Li}^{38}$ employed the QCT method to study the vector correlations between the product and reagent for the title reaction occurred on the $1 \mathrm{~A}^{\prime} \mathrm{DK}$ PES. They found that the $\mathrm{OH}$ products are produced mainly in the plane of H-O-H plane. In addition, Chen et al. ${ }^{39}$ studied the vector correlation of this reaction on the same PES at collision energy of $19 \mathrm{kcal} / \mathrm{mol}$ but with the variation of vibration quantum number $\mathbf{v}$. They concluded that the product alignment and state distribution are sensitive to the vibration quantum number. In turn, Liu et $a l^{40}$ calculated the dynamics of the reactions $\mathrm{O}\left({ }^{1} \mathrm{D}\right)+\mathrm{H}_{2}, \mathrm{D}_{2}$ and HD using the QCT method on the same PES. Their results show that the intermediate geometrical structures and lifetimes of the reactive collisions play a vital role in these reactions.

The most accurate theoretical calculation of gas-phase bimolecular reaction is through quantum dynamics approach based on scattering theory. After a systematic analysis, Ju et al. ${ }^{41}$ proved that for most reactions QCT results are consistent with quantum ones. A good agreement of the QCT results with quantum results for the $\mathrm{O}\left({ }^{1} \mathrm{D}\right)+\mathrm{H}_{2}$ reaction had been found by $\mathrm{Li},{ }^{38}$ which enhances the use of quasiclassical trajectory method in this study.

To shed more light on stereodynamics of the title reaction, we report the results of QCT calculation for the reaction $\mathrm{O}\left({ }^{1} \mathrm{D}\right)+$ $\mathrm{H}_{2} \rightarrow \mathrm{OH}+\mathrm{H}$ which are focused on vector correlation properties including the polarization-dependent differential cross sections, product rotational alignment and scattering direction. In this paper, our calculation of the vector correlations between products and reagents for this reaction is performed on the $1 \mathrm{~A}^{\prime}$ DK PES. This potential energy surface is constructed by Dobbyn and Knowles with high level ab initio calculation for the $1^{1} \mathrm{~A}^{\prime}$, $1^{1} \mathrm{~A}^{\prime \prime}$ and $2^{1} \mathrm{~A}^{\prime}$ electronic states and the electronic coupling between the $1^{1} \mathrm{~A}^{\prime}$ and $2 \mathrm{~A}^{\prime} .{ }^{1}$ We choose the trajectory initiated with the hydrogen molecule in the $\mathbf{v}=0$ and $\mathbf{j}=0$-5 levels, and the collision energy is $23.06 \mathrm{kcal} / \mathrm{mol}(1 \mathrm{eV})$. In the calculation, a batch of 50000 trajectories is run and the integration step is chosen to be $0.1 \mathrm{fs}$. The present work is organized as follows.
Section 2 briefly introduces the stereodynamics calculation method. Section 3 presents the calculation results and underlines the discussions about them. Finally, the conclusion is gathered in Section 4.

\section{Theoretical Method}

Fig. 1 shows the center of-mass coordinate system used to describe the $\mathbf{k}, \mathbf{k}^{\prime}, \mathbf{j}^{\prime}$ correlations. $\theta$ is the angle between the reagent relative velocity and product relative velocity. $\theta_{r}$ and $\phi_{r}$ are the polar and azimuthal angles of final rotational momentum j'.

The fully correlated center-of-mass angular distribution is written as the sum

$$
\begin{aligned}
& P\left(\cos \theta, \cos \theta_{r}, \phi_{r}\right) \\
& \quad=\frac{1}{4 \pi} \sum_{k=0}^{\infty} \sum_{q=-k}^{k}(2 k+1) \times \frac{2 \pi}{\sigma} \frac{d \sigma_{k q}}{d \omega} C_{k q}\left(\theta_{r}, \phi_{r}\right)
\end{aligned}
$$

Where $\omega=\theta, \phi$ and $\omega_{r}=\theta_{r}, \phi_{r}$ refers to the coordinates of the unit vectors $\mathbf{k}^{\prime}$ and $\mathbf{j}^{\prime}$ along the directions of the product relative velocity and rotational angular momentum vectors in the CM frame, respectively. $\frac{1}{\sigma} \frac{d \sigma_{k q}}{d \omega}$ is the generalized polarization dependent differential cross-sections(PDDCS). ${ }^{42-43}$

The PDDCSs are written as follow: ${ }^{38,44-46}$

$\frac{2 \pi}{\sigma} \frac{d \sigma_{k 0}}{d \omega}=0 \quad$ when $\mathrm{k}$ is odd

$\frac{2 \pi}{\sigma} \frac{d \sigma_{k q+}}{d \omega}=\frac{2 \pi}{\sigma} \frac{d \sigma_{k q}}{d \omega}+\frac{2 \pi}{\sigma} \frac{d \sigma_{k-q}}{d \omega}=0$ when $\mathrm{k}$ is even, $q$ is odd or $k$ is odd $q$ is even

$\frac{2 \pi}{\sigma} \frac{d \sigma_{k q-}}{d \omega}=\frac{2 \pi}{\sigma} \frac{d \sigma_{k q}}{d \omega}-\frac{2 \pi}{\sigma} \frac{d \sigma_{k-q}}{d \omega}=0$ when $k$ is even, $q$ is odd or $k$ is odd $q$ is even

We also can summarize it as

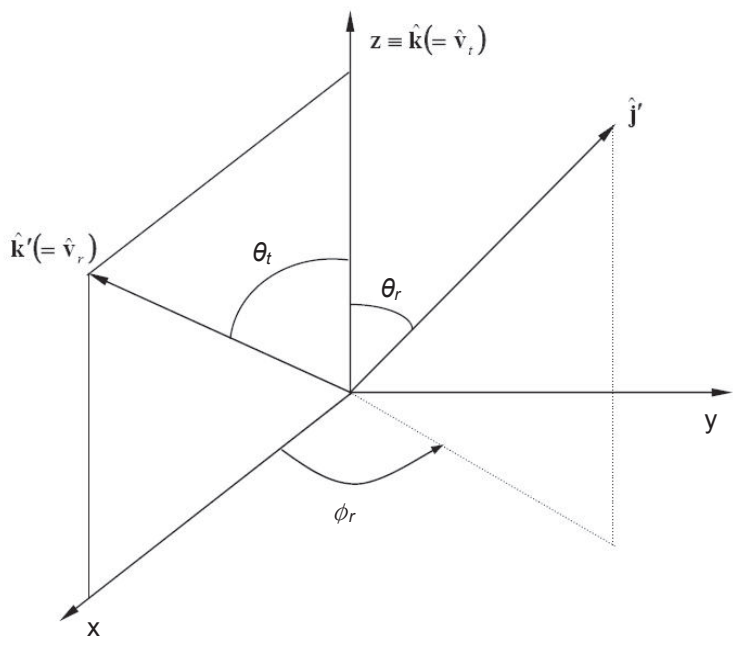

Figure 1. The center-of-mass coordinate system used to describe the $\mathbf{k}, \mathbf{k}^{\prime}, \mathbf{j}^{\prime}$ correlations. 


$$
\frac{2 \pi}{\sigma} \frac{d \sigma_{k q \pm}}{d \omega}=\sum_{k_{1}} \frac{\left[k_{1}\right]}{4 \pi} S_{k q \pm}^{k_{1}} C_{k_{1}-q}(\theta, 0)
$$

And $S_{q \pm}^{k_{1}}$ was expressed as

$$
\boldsymbol{S}_{k q \pm}^{k_{1}}=\left\langle c_{k_{1 q}}(\theta, 0) c_{k q}\left(\theta_{r}, 0\right)\left[(-1)^{q} e^{i q \phi_{r}} \pm e^{-i q \phi_{r}}\right]\right\rangle
$$

Two vector correlations $\left(\mathbf{k}-\mathbf{j}^{\prime}, \mathbf{k}-\mathbf{k}^{\prime}\right.$, or $\left.\mathbf{k}^{\prime}-\mathbf{j}^{\prime}\right)$ can be expanded into a series of Legendre polynomials. ${ }^{47-48}$ The $P\left(\theta_{r}\right)$ distribution corresponding to the $\mathbf{k}-\mathbf{j}$ ' correlation is written as

$$
P\left(\theta_{r}\right)=\frac{1}{2} \sum_{k}[k] a_{0}^{k} P_{k}\left(\cos \theta_{r}\right)
$$

The $a_{0}^{k}$ coefficients (polarization parameters) are given by $a_{0}^{k}=\left\langle P_{k}\left(\cos \theta_{r}\right)\right\rangle$

The brackets represent an average over all the reactive trajectories. The dihedral angle distribution of the $\mathbf{k}-\mathbf{k}^{\prime}-\mathbf{j}^{\prime}$ three vectors correlation is characterized by the angle $P\left(\phi_{r}\right)$. The distribution of the dihedral angle $P\left(\phi_{r}\right)$ could be expanded as a Fourier series

$$
P\left(\phi_{r}\right)=\frac{1}{2 \pi}\left(1+\sum_{n \text { even } \geq 2} a_{n} \cos n \phi_{r}+\sum_{n} b_{n} \sin \phi_{r}\right)
$$

With $a_{n}$ and $b_{n}$ given by

$$
\begin{aligned}
& a_{n}=2\left\langle\cos n \phi_{r}\right\rangle \\
& b_{n}=2\left\langle\sin n \phi_{r}\right\rangle
\end{aligned}
$$

In the work, when $P\left(\phi_{r}\right)$ is expended up to $\mathrm{n}=24$, the results are converged.

The joint of probability density function of angles $\theta_{r}$ and $\phi_{r}$, which define the direction of $\mathbf{j}^{\prime}$, can be written as

$$
\begin{aligned}
& P\left(\theta_{r}, \phi_{r}\right)=\frac{1}{4 \pi} \sum_{k q}[k] a_{q}^{k} C_{k q}\left(\theta_{r}, \phi_{r}\right)^{*} \\
& \quad=\frac{1}{4 \pi} \sum_{k} \sum_{q \geq 0}\left[a_{q \pm}^{k} \cos q \phi_{r}-a_{q \mp}^{k} i \sin q \phi_{r}\right] C_{k q}\left(\theta_{r}, 0\right)
\end{aligned}
$$

The parameter is evaluated as

$$
\begin{aligned}
& a_{q \pm}^{k}=2\left\langle C_{k|q|}\left(\theta_{r}, 0\right) \cos q \phi_{r}\right\rangle \quad k \text { even } \\
& a_{q \pm}^{k}=2 i\left\langle C_{k|q|}\left(\theta_{r}, 0\right) \sin q \phi_{r}\right\rangle \quad k \text { odd }
\end{aligned}
$$

During the calculation, $P\left(\theta_{r}, \phi_{r}\right)$ is expanded up to $k=7$, which is sufficient for good convergence.

\section{Results and Discussion}

PDDCSs can be used to describe the correlation of $\mathbf{k}-\mathbf{k}^{\prime}-\mathbf{j}^{\prime}$ and scattering direction of product molecule. The calculated results of PDDCSs for the $\mathrm{O}\left({ }^{1} \mathrm{D}\right)+\mathrm{H}_{2} \rightarrow \mathrm{OH}+\mathrm{H}(\mathbf{v}=0, \mathbf{j}=0-5)$ reaction at a collision energy of $23.06 \mathrm{kcal} / \mathrm{mol}$ are shown in Fig. 2. The $(2 \pi / \sigma)\left(d \sigma_{00} / d \omega_{t}\right)$ is a simple differential cross section (DCS), which only describes the $\mathbf{k}-\mathbf{k}^{\prime}$ correlation or the scattering direction of the product, and it is not associated with the alignment or orientation of the product rotational angular momentum vector. As can be seen from Fig. 2(a-f), all the calculated PDDCSs $(2 \pi / \sigma)\left(d \sigma_{00} / d \omega_{t}\right)$ show a forward-backward asymmetry with a biased forward scattering. It is known that PDDCS $(2 \pi / \sigma)\left(d \sigma_{20} / d \omega_{t}\right)$ represents the expectation value of the second Legendre moment $\left\langle P_{2}(\cos \theta)\right\rangle$. As can be found from the distributions of PDDCS $(2 \pi / \sigma)\left(d \sigma_{20} / d \omega_{t}\right)$, $\mathbf{j}^{\prime}$ is preferentially polarized along the direction perpendicular to $\mathbf{k}$ at $\theta_{t}=0^{\circ}$ and $\theta_{t}=180^{\circ}\left(\theta_{t}\right.$ is the angle between $\mathbf{k}$ and $\left.\mathbf{k}^{\prime}\right)$, since it has the most negative values of -0.3 and -0.25 at these two angles. However, the PDDCS $(2 \pi / \sigma)\left(d \sigma_{20} / d \omega_{t}\right)$ seems nearly isotropic side ways scattering at other scattering angles. This means the alignment effect is weaker when the products of $\mathrm{OH}$ are produced not at the scattering angles $\theta_{t}=0^{\circ}$ and $180^{\circ}$. Generally, the well in the PES can lead to a weak product rotational alignment effect, because it may lead to 'loss' of memory for the angular momentum alignment in that the separation of the products will take various directions in space. Another QCT calculation ${ }^{48}$ of PDDCS $(2 \pi / \sigma)\left(d \sigma_{20} / d \omega_{t}\right)$ for the same reaction based on the SL1 (Schineke-Lester) PES also presented the same strong product alignment for $\theta_{t}=0^{\circ}$ and $180^{\circ}$. The calculation also showed that the PDDCS $(2 \pi / \sigma)\left(d \sigma_{20} / d \omega_{t}\right)$ becomes nearly isotropic for sideways scattering, and supported the results of our work. It can be seen from Figure 2 that all PDDCSs with $\mathrm{q} \neq 0$ are equal to zero at $q_{t}=0^{\circ}, 180^{\circ}$ and the PDDCSs $(2 \pi / \sigma)\left(d \sigma_{22+} / d \omega_{t}\right)$ are nonzero at scattering angles away from these two angles. The values of PDDCSs $(2 \pi / \sigma)\left(d \sigma_{21-} / d \omega_{t}\right)$ which are related to $<\sin ^{2} \theta_{r}, \cos ^{2} \phi_{r}>$, are close to zero, that is to say, $(2 \pi / \sigma)\left(d \sigma_{21-} / d \omega_{t}\right)$ is obviously independent of the variation of j. $(2 \pi / \sigma)\left(d \sigma_{22+} / d \omega_{t}\right)$ and $(2 \pi / \sigma)\left(d \sigma_{21-} / d \omega_{t}\right)$ show the anisotropy of production scattering distribution. Since the values of $(2 \pi / \sigma)\left(d \sigma_{22+} / d \omega_{t}\right)$, $(2 \pi / \sigma)\left(d \sigma_{21-} / d \omega_{t}\right)$ are relatively very small for all the angles, they can not provide much information about the reaction mechanism. In addition, Fig. 2 clearly shows that the four shapes of calculated PDDCSs are displayed similarly with different $\mathbf{j}$. Thus, it can be drawn the conclusion that PDDCSs are rather insensitive to the rotation quantum number $\mathbf{j}$ on this singlet state.

However, there are still very slight differences in the calculated results with different $\mathbf{j}$. The angular distributions of PDDCS $(2 \pi / \sigma)\left(d \sigma_{00} / d \omega_{t}\right)$ and PDDCS $(2 \pi / \sigma)\left(d \sigma_{20} / d \omega_{t}\right)$ are amplified in Fig. 3. It can be found in Fig. 3(a), as rotation quantum number $\mathbf{j}$ increases, degree of forward scattering increases slightly whereas that of backward scattering decreases slightly. Fig. 3(b) shows that the values of PDDCS $(2 \pi / \sigma)\left(d \sigma_{20} / d \omega_{t}\right)$ decreases slightly with increasing $\mathbf{j}$ for scattering angles smaller than about $125^{\circ}$ while vice versa for $\theta_{t}>125^{\circ}$.

As already emphasized above, PDDCSs shown in Fig. 2 and Fig. 3 are stereodynamical quantities amenable to direct experimental determination and they could provide enough information about the $\mathbf{k}-\mathbf{k}^{\prime} \mathbf{- j}$ ' correlation as well as the angular moment- 
(a)

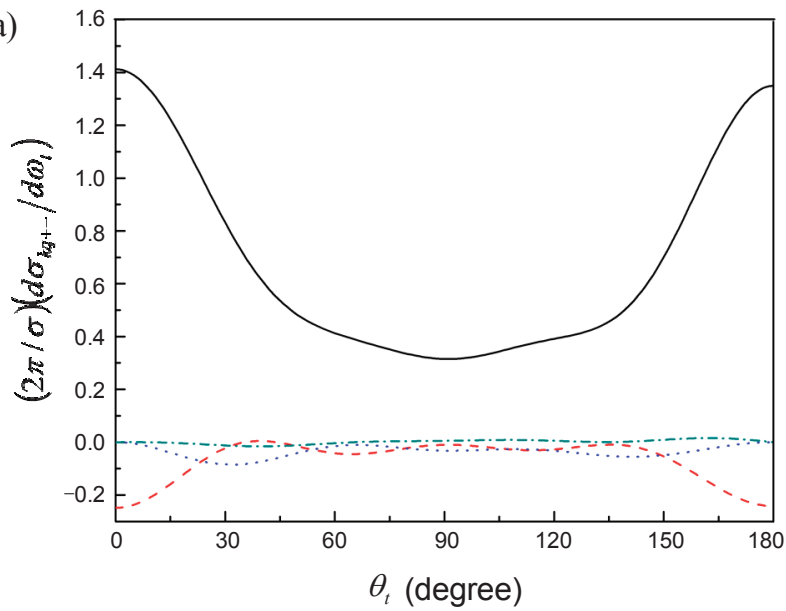

(c)

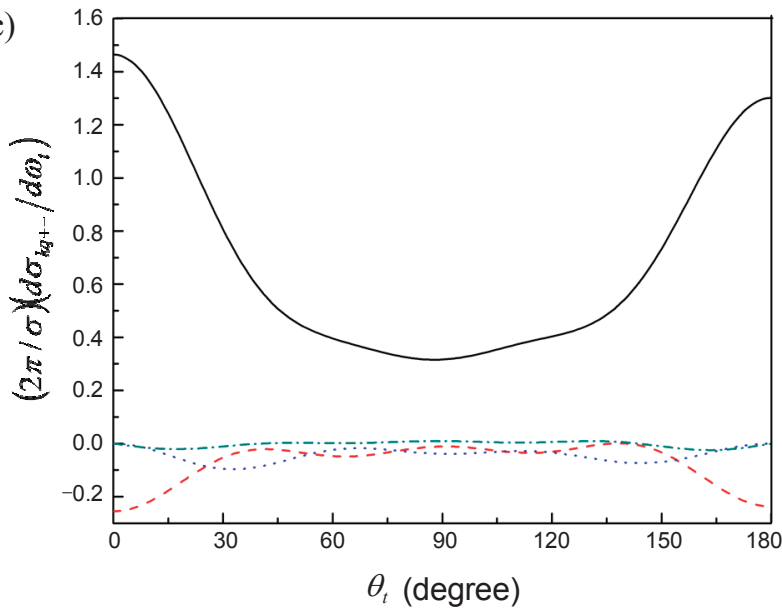

(e)

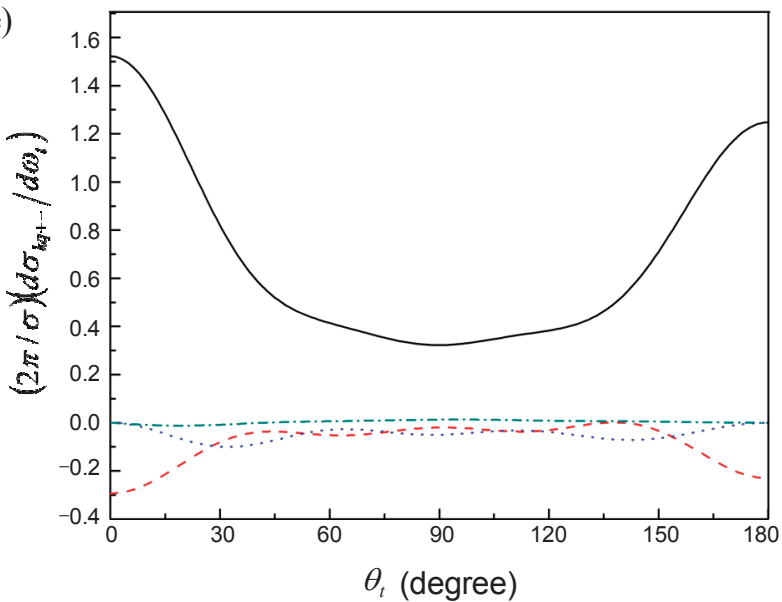

(b)

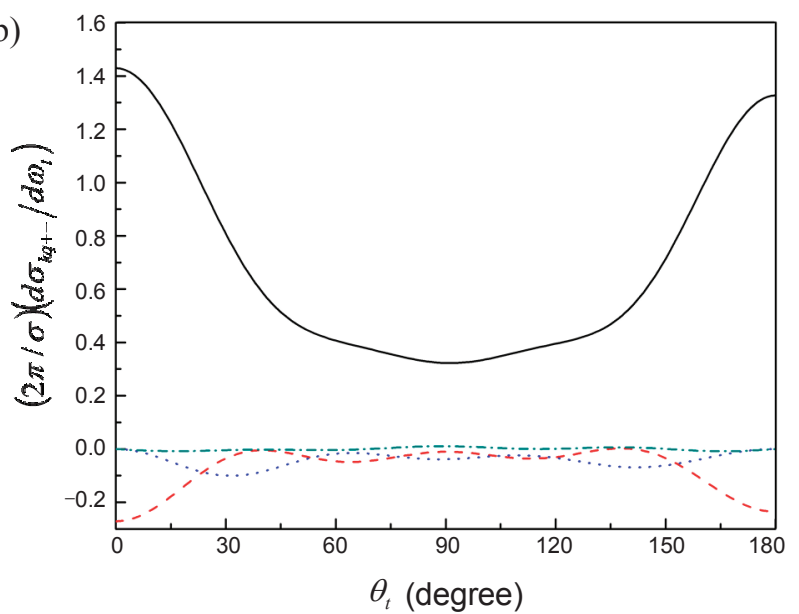

(d)

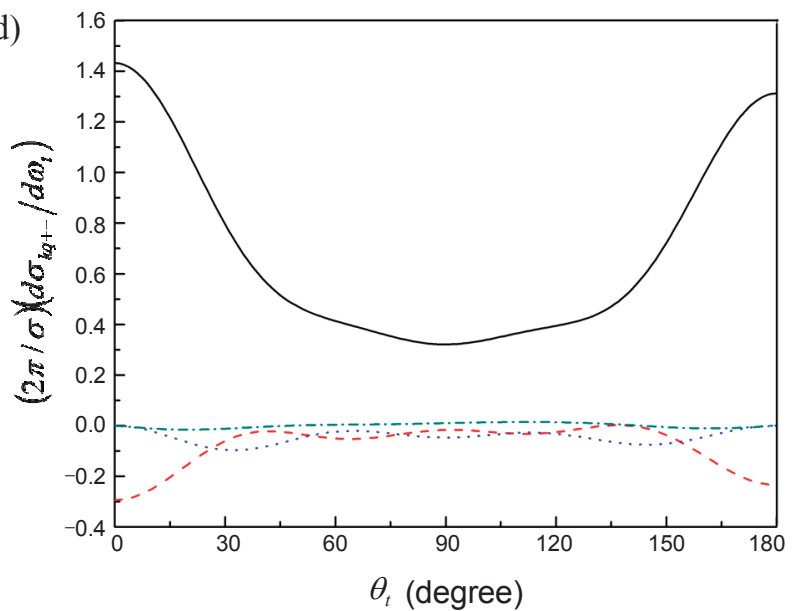

(f)

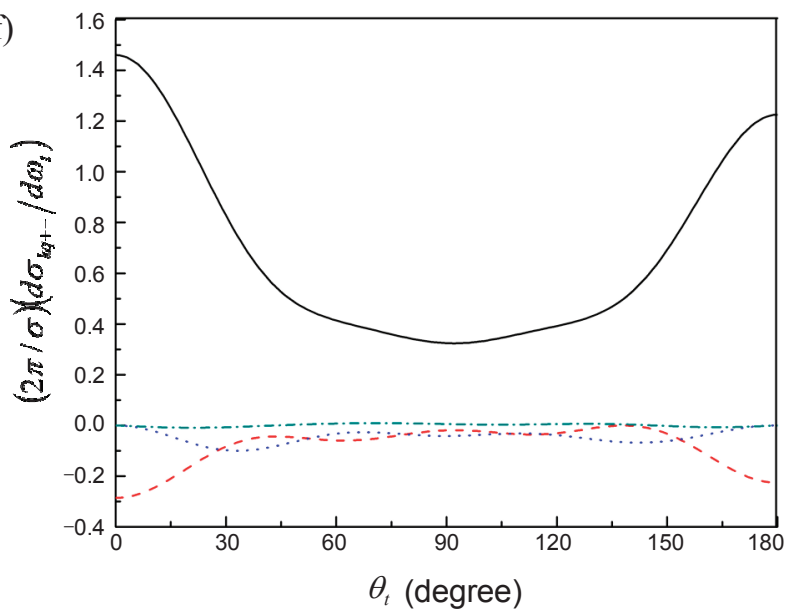

Figure 2. Four polarization dependent differential cross-sections of the reaction $\mathrm{O}\left({ }^{1} \mathrm{D}\right)+\mathrm{H}_{2} \rightarrow \mathrm{OH}+\mathrm{H}(\mathbf{v}=0, \mathbf{j}=0-5)$ at the collision energy of 23.06 $\mathrm{kcal} / \mathrm{mol}$. (a) $\mathbf{j}=0$, (b) $\mathbf{j}=1$, (c) $\mathbf{j}=2$, (d) $\mathbf{j}=3$, (e) $\mathbf{j}=4$, (f) $\mathbf{j}=5$. Solid line indicates $(2 \pi / \sigma)\left(d \sigma_{00} / d \omega_{t}\right)$, dash line indicates $(2 \pi / \sigma)\left(d \sigma_{20} / d \omega_{t}\right)$, dot line indicates $(2 \pi / \sigma)\left(d \sigma_{22+} / d \omega_{t}\right)$, dash dot line indicates $(2 \pi / \sigma)\left(d \sigma_{21-} / d \omega_{t}\right)$.

um polarization. But PDDCSs are not very intuitive. For the purpose of getting a better graphical representation of polarization for the $\mathrm{OH}$ product from the $\mathrm{O}\left({ }^{1} \mathrm{D}\right)+\mathrm{H}_{2} \rightarrow \mathrm{OH}+\mathrm{H}$ reaction, we have plotted the distributions of $P\left(\theta_{r}\right)$ and $P\left(\phi_{r}\right)$, which are shown in Fig. 4 and Fig. 5, respectively. Fig. 4 clearly illustrates that the distributions of $P\left(\theta_{r}\right)$ peak at $q_{r}$ angles close to $90^{\circ}$ and are symmetric with respect to $90^{\circ}$ due to the planar symmetry of the system. Therefore, The distribution of $P\left(\theta_{r}\right)$, describing the $\mathbf{k}$, j' correlation, indicates that the product angular momentum $\mathbf{j}$ ' tends to align along the direction perpendicular to $\mathbf{k}$. It can be noted from Fig. 4 that the discrepancy between the distributions of $P\left(\theta_{r}\right)$ with different value of $\mathbf{j}$ is not very obvious. As the 
(a)

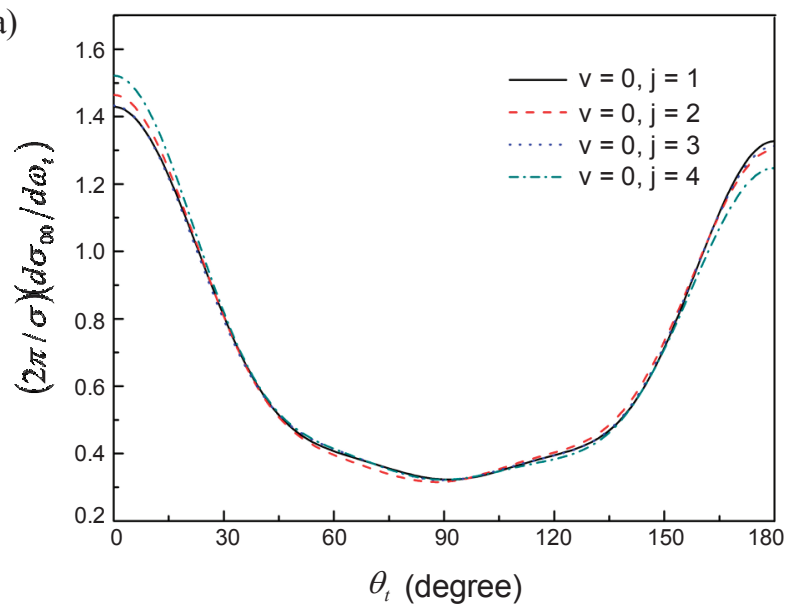

(b)

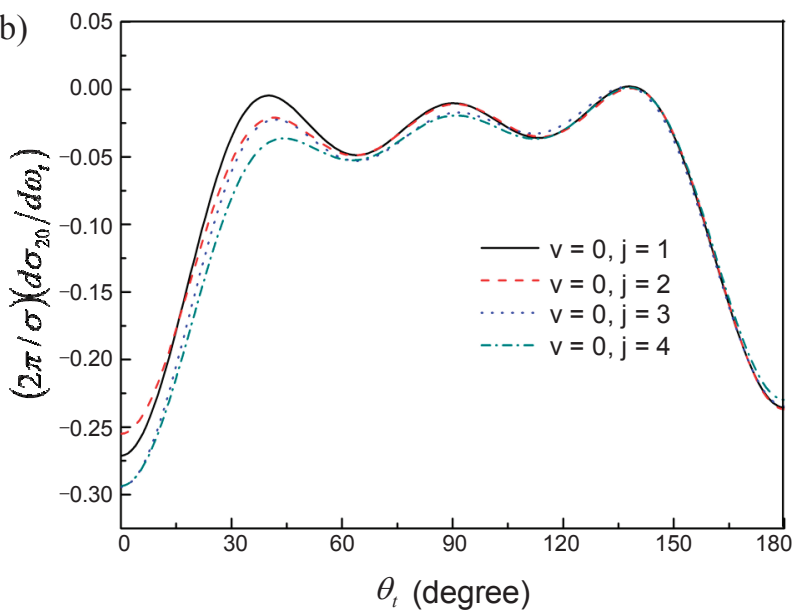

Figure 3. Two calculated PDDCSs $(2 \pi / \sigma)\left(d \sigma_{00} / d \omega_{t}\right)$ and $(2 \pi / \sigma)\left(d \sigma_{20} / d \omega_{t}\right)$ of reaction $\mathrm{O}\left({ }^{1} \mathrm{D}\right)+\mathrm{H}_{2} \rightarrow \mathrm{OH}+\mathrm{H}$ at the same collision energy. (a) $\operatorname{PDDCS}(2 \pi / \sigma)\left(d \sigma_{00} / d \omega_{t}\right)$; (b) $\operatorname{PDDCS}(2 \pi / \sigma)\left(d \sigma_{20} / d \omega_{t}\right)$.

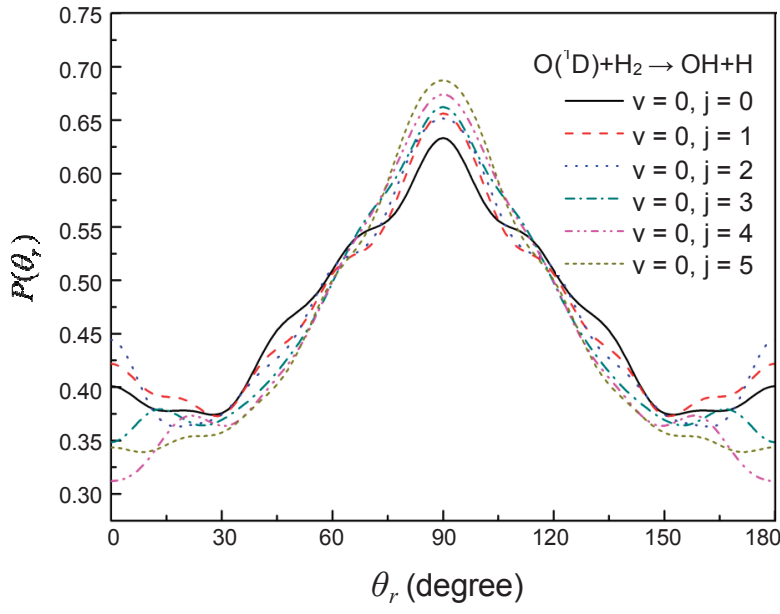

Figure 4. The distribution of $P\left(\theta_{r}\right)$ reflecting the $\mathbf{k}-\mathbf{j}$ ' correlation at the collision energy of $23.06 \mathrm{kcal} / \mathrm{mol}$ for the $\mathrm{O}\left({ }^{1} \mathrm{D}\right)+\mathrm{H}_{2} \rightarrow \mathrm{OH}+\mathrm{H}$ reactions.

rotation quantum number $\mathbf{j}$ rises, there is a contraction in the calculated $P\left(\theta_{r}\right)$ distributions with the peak moving a little upward. Thus, it can be concluded that the product polarization becomes stronger with the increasing of $\mathbf{j}$, but the trend is not so obvious.

Because $\phi_{r}$ defines the dihedral angle between the planes consisting of $\mathbf{k}-\mathbf{k}^{\prime}$ and $\mathbf{k}-\mathbf{j}^{\prime}$, the $P\left(\phi_{r}\right)$ distribution correlates to the $\mathbf{k}-\mathbf{k}^{\prime}-\mathbf{j}^{\prime}$ vector correlation and can provide stereodynamical information on both product alignment and orientation. The dihedral angle distributions of $P\left(\phi_{r}\right)$ for the $\mathrm{O}\left({ }^{1} \mathrm{D}\right)+\mathrm{H}_{2} \rightarrow$ $\mathrm{OH}+\mathrm{H}$ reaction are presented in Fig. 5. It expresses the correlation of the product rotation angular momentum vectors and velocity of reagents and products. Clearly, the $P\left(\phi_{r}\right)$ distributions demonstrate a dependent behavior on rotation quantum number $\mathbf{j}$. Despite these noticeable differences associated with $\mathbf{j}$ for which we will discuss later, there still exists a common feature for the $P\left(\phi_{r}\right)$ distributions in that they tend to be asymmetric about $\phi_{r}=180^{\circ}$ with respect to the scattering $\mathbf{k}-\mathbf{k}^{\prime}$ plane, with two different peaks appearing at/around $\phi_{r}=90^{\circ}$ and

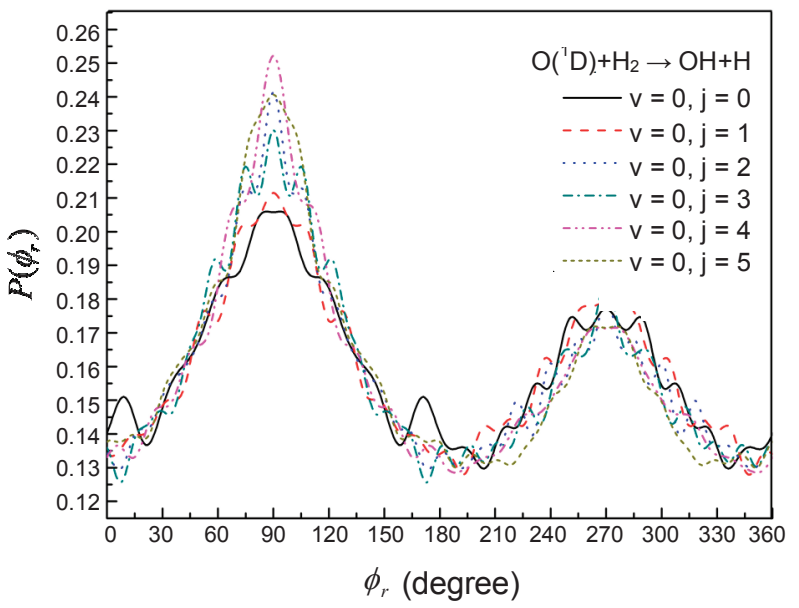

Figure 5. The dihedral angle distribution of $\mathbf{j}^{\prime}, P\left(\phi_{r}\right)$ with respect to the $\mathbf{k}-\mathbf{k}^{\prime}$ plane at the collision energy of $23.06 \mathrm{kcal} / \mathrm{mol}$.

$\phi_{r}=270^{\circ}$, respectively. This feature gives the information that products tend to align along the direction of y-axis that is perpendicular to the scattering plane. It may because the ground state $\mathrm{H}_{2} \mathrm{O}$ molecule has two lone pairs of electrons that are nearly perpendicular to the plane formed by the two bond pairs of electrons. The lone pairs of electrons will repel the $\mathrm{O}-\mathrm{H}$ bond from two sides perpendicular to the $\mathrm{H}-\mathrm{O}-\mathrm{H}$ plane. So the $\mathrm{OH}$ products are mainly produced in the $\mathrm{H}-\mathrm{O}-\mathrm{H}$ plane. And the reason of the asymmetric $P\left(\phi_{r}\right)$ distribution is probably that the repulsive energy between $\mathrm{H}-\mathrm{H}$ atoms leads to the violation of the symmetry when the reaction occurs. We can see that the peak at $\phi_{r}=270^{\circ}$ is far smaller than at $\phi_{r}=90^{\circ}$, which is connected to the fact that the rotational angular momentum vector of the $\mathrm{OH}$ product is preferentially oriented along the positive direction of y-axis. The peaking at $\phi_{r}=90^{\circ}$ also implies a preference for right-handed $\mathrm{OH}$ rotation in planes parallel to the scattering plane. We will use the term 'in-plane' to refer to this preference of product molecule rotation in planes parallel to the scattering plane, and the term 'out-of-plane' to refer to the preference of 
(a)

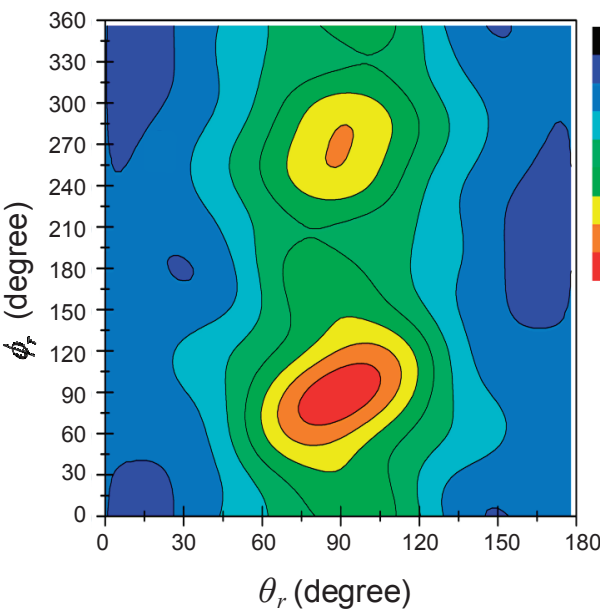

(c)

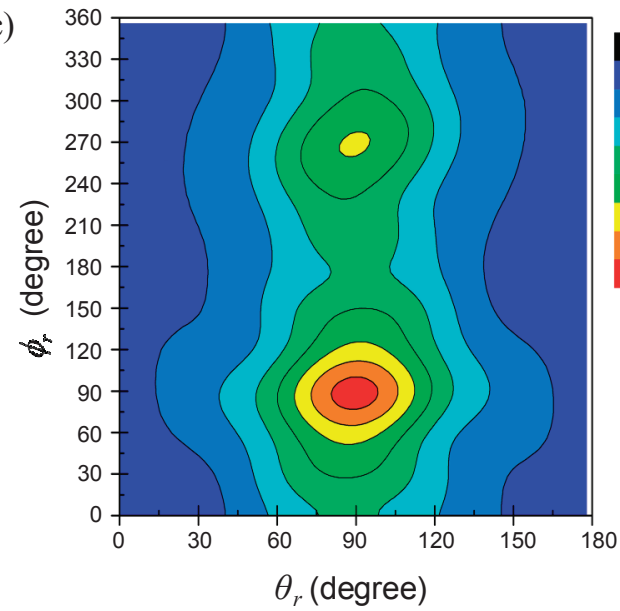

(e)

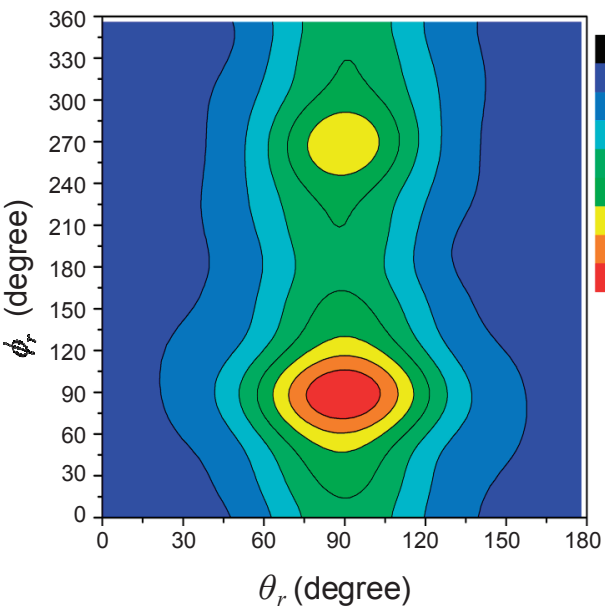

(b)

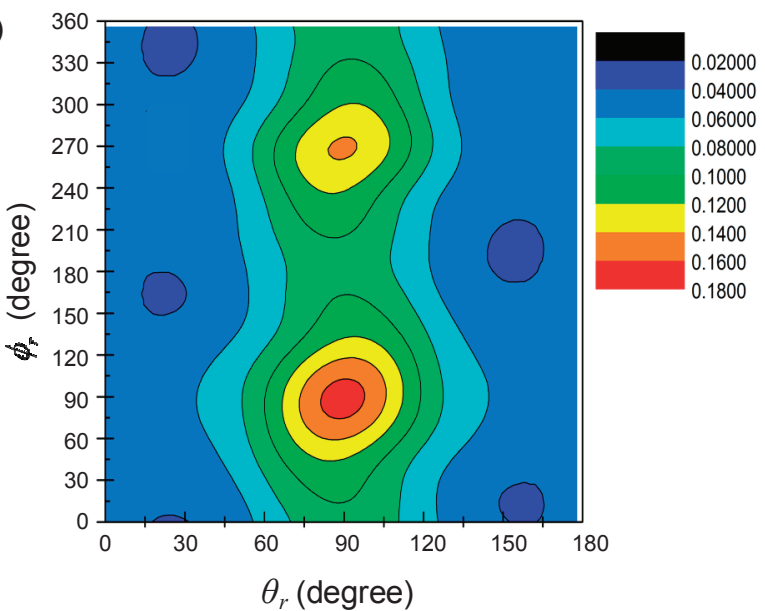

(d)

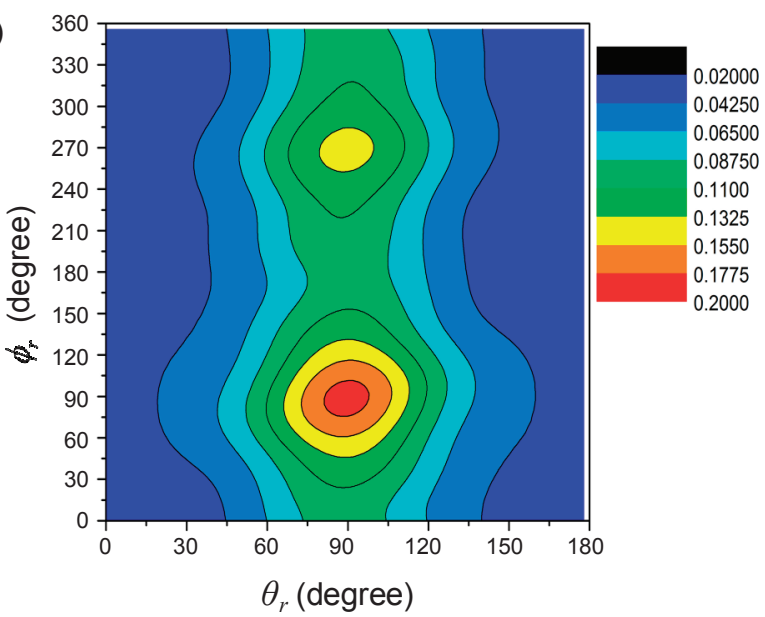

(f)

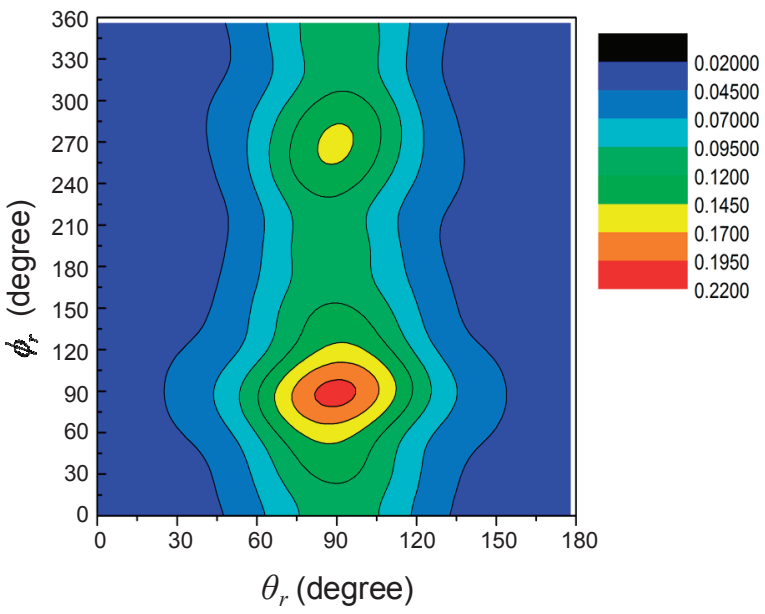

Figure 6. Polar plots of $P\left(\theta_{r}, \phi_{r}\right)$ distribution averaged over all scattering angles at the collision energy of $23.06 \mathrm{kcal} / \mathrm{mol}$ of the reaction $\mathrm{O}\left({ }^{\mathrm{I}} \mathrm{D}\right)+\mathrm{H}_{2} \rightarrow \mathrm{OH}+\mathrm{H}(\mathbf{v}=0, \mathbf{j}=0-5)$. (a) $\mathbf{j}=0$, (b) $\mathbf{j}=1$, (c) $\mathbf{j}=2$, (d) $\mathbf{j}=3$, (e) $\mathbf{j}=4$, (f) $\mathbf{j}=5$.

product molecule in planes perpendicular to the scattering plane.

Comparing the $P\left(\phi_{r}\right)$ distributions generated on the five different values of $\mathbf{j}$, it can be concluded that the orientation peak of product molecules becomes stronger when rotation quantum number $\mathbf{j}$ rises from 0 to 5 , because the $\phi_{r}=90^{\circ}$ peak and $\phi_{r}=$ $270^{\circ}$ peak show an upward trend and reduction trend with the increasing of $\mathbf{j}$. That is, the $P\left(\phi_{r}\right)$ distribution on this singlet state is found to be dependent to the rotation quantum number $\mathbf{j}$ and this reaction has a strong preference for the product molecules rotating 'in-plane'.

The variation in the dihedral angular distribution implies the rotation quantum number $\mathbf{j}$ plays an important role in dynamical stereochemistry of the reaction $\mathrm{O}\left({ }^{1} \mathrm{D}\right)+\mathrm{H}_{2} \rightarrow \mathrm{OH}+\mathrm{H}$. 
Fig. 6 depicts the angular momentum polarization in the form of a polar plot of the distribution $P\left(\theta_{r}, \phi_{r}\right)$, which represents the scattering angle average of the distribution. As can be seen from Fig. 6, the distributions of $P\left(\theta_{r}, \phi_{r}\right)$ which peak at $\left(90^{\circ}\right.$, $\left.90^{\circ}\right)$ and $\left(90^{\circ}, 270^{\circ}\right)$ are in good agreement with the distributions of $P\left(\theta_{r}\right)$ and $P\left(\phi_{r}\right)$ of the products. The distributions of $P\left(\theta_{r}, \phi_{r}\right)$ also support that $\mathrm{OH}$ products are preferentially polarized perpendicular to the scattering plane and the reaction is dominated by 'in-plane' mechanisms.

Chen et al. ${ }^{39}$ studied the reaction $\mathrm{O}\left({ }^{1} \mathrm{D}\right)+\mathrm{H}_{2}(\mathbf{v}=0-3, \mathbf{j}=0) \rightarrow$ $\mathrm{OH}+\mathrm{H}$ on the same PES. They also found that the forward scattering trend gets stronger while its backward scattering trend gets weaker with increasing the vibration quantum number $\mathbf{v}$. A comparison between the calculated PDDCSs from the two studies revealed that the reduction trend of the backward scattering is more obvious in Chen et al.'s study with respect to the variation of $\mathbf{v}$. Comparing the $P\left(\theta_{r}\right)$ and $P\left(\phi_{r}\right)$ distributions from both calculations, we found that the product alignment and orientation are more sensitive to initial vibration quantum number $\mathbf{v}$ than rotation quantum number $\mathbf{j}$, since the variation of the two distributions with $\mathbf{v}$ are found to be more conspicuous in their study than in the present study.

\section{Conclusion}

In this work, the reaction $\mathrm{O}\left({ }^{1} \mathrm{D}\right)+\mathrm{H}_{2}(\mathbf{v}=0, \mathbf{j}=0-5) \rightarrow \mathrm{OH}+\mathrm{H}$ has been theoretically studied using quasiclassical trajectory (QCT) method on the DK potential energy surface at a collision energy of $23.06 \mathrm{kcal} / \mathrm{mol}$. Stereodynamics information was obtained for different initial rotational state of reactant $\mathrm{H}_{2}$. The four normalized PDDCSs have been calculated and the results indicate the reaction mainly shows both forward and backward scattering. With increasing the value of $\mathbf{j}$, the product forward scattering is slightly favored. The distribution of $P\left(\theta_{r}\right)$ indicates that the product is strongly polarized and the distribution of $P\left(\phi_{r}\right)$ shows the rotational angular momentum vector of the $\mathrm{OH}$ product is preferentially oriented along the positive direction of y-axis. We have also compared the distributions of $P\left(\theta_{r}\right), P\left(\phi_{r}\right)$ and $P\left(\theta_{r}, \phi_{r}\right)$ of the title reaction for different j. The distribution of $P\left(\phi_{r}\right)$ shows a dependence on $\mathbf{j}$, with the product orientation peaks are being found to become stronger with increasing the value of $\mathbf{j}$. However, the discrepancy between the distributions of $P\left(\theta_{r}\right)$ with different value of $\mathbf{j}$ is not so obvious. Comparison with Chen's results has revealed that the product alignment and orientation are more sensitive to $\mathbf{v}$ than $\mathbf{j}$ in this reaction.

Although these results were obtained only on the $1 \mathrm{~A}^{\prime} \mathrm{PES}$, they still reveal some new aspects about the title reaction. Future accurate calculations and experiments are still needed to unravel the mechanism of this reaction. We hope that this calculation may provide experimentalists with some help when they interpret their experimental data.

Acknowledgments. The authors acknowledge Professor Keli Han for providing the QCT code of stereodynamics, as well as some precious advice.

\section{References}

1. Chu, T. S.; Han, K. L. Phys. Chem. Chem. Phys. 2008, 10, 2431.

2. Schinke, R.; Lester, W. A. J. Chem. Phys. 1980, 72, 3754.

3. Kuntz, P. J.; Niefer, B. I.; Sloan, J. J. J. Chem. Phys. 1988, 88, 3629.

4. Schatz, G. C.; Aioannou, A.; Pederson, L. A.; Harding, L. B.; Hollebeek, T.; Ho, T. S.; Rabitz, H. J. Chem. Phys. 1997, 107, 2340.

5. Butler, J. E.; Jursich, G. M.; Waston, I. A.; Wisenfeld, J. R. J. Chem. Phys. 1986, 84, 5365.

6. Pierce, B. M.; Bennett, J. A.; Birge, R. R. J. Chem. Phys. 1982, 77,6343

7. Fitzcharles, M. S.; Schatz, G. C. J. Phys. Chem. 1986, 90, 3634.

8. Alexander, A. J.; Aoiz, F. J.; Brouard, M.; Simons, J. P. Chem. Phys. Lett. 1996, 256, 561.

9. Badenhoop, K.; Koizumi, K.; Schatz, G. C. J. Chem. Phys. 1989, 91, 142.

10. Zhao, G. J.; Liu, J. Y.; Zhou, L. C.; Han, K. L. J. Phys. Chem. B 2007, 111, 8940.

11. Alagia, M.; Balucani, N.; Cartechini, L.; Casavecchia, P.; Volpi, G. G.; Kuntz, P. J.; Sloan, J. J. J. Chem. Phys. 1998, 108, 6698.

12. Ahmed, M.; Peterka, D. S.; Suits, A. Chem. Phys. Lett. 1999, 301, 372 .

13. Hsu, Y. T.; Liu, K. J. Chem. Phys. 1997, 107, 1664.

14. Dobbyn, A. J.; Knowles, P. J. Faraday Discuss. 1998, 110, 247.

15. Liu, X.; Lin, J. L.; Harich, S.; Yang, X. J. Chem. Phys. 2000, $113,1325$.

16. Ho, T. S.; Hollebeek, T.; Rabitz, H.; Harding, L. B.; Schatz, G. C. J. Chem. Phys. 1996, 105, 10472.

17. Dobbyn, A. J.; Knowles, P. J. Mol. Phys. 1997, 91, 1107

18. Chu, T. S.; Zhang, X.; Han, K. L. J. Chem. Phys. 2005, 122, 214301.

19. Hsu, Y. T.; Liu, K.; Pederson, L. A.; Schatz, G. C. J. Chem. Phys. 1999, 111, 7931

20. Koppe, S.; Laurent, T.; Naik, P. D.; Volpp, H. R.; Wolfrum, J.; Arusi-Parpar, T.; Bar, I.; Rosenwaks, S. Chem. Phys. Lett. 1993, 214,546

21. Chu, T. S.; Zhang, Y.; Han, K. L. Int. Rev. Phys. Chem. 2006, 25 , 201.

22. Althorpe, S. C.; Clary, D. C. Annu. Rev. Phys. Chem. 2003, 54, 493.

23. Song, J. B.; Gislason, E. A. Chem. Phys. 1996, 202, 1.

24. Johnson, B. R.; Winter, N. W. J. Chem. Phys. 1977, 66, 4116.

25. Zhao, G. J.; Han, K. L. Biophys. J. 2008, 94, 38.

26. Fano, U.; J. Macek, H. Rev. Mod. Phys. 1973, 45, 553.

27. Baenwell, J. D.; Loeser, J. G.; Herschbach, D. R. J. Phys. Chem. 1983, 87, 2781.

28. Han, K. L.; He, G. Z.; Lou, N. Q. J. Chem. Phys. 1996, 105, 8699.

29. Zhao, G. J.; Han, K. L.; Lei, Y. B.; Dou, Y. J. Chem. Phys. 2007, $127,094307$.

30. Zhang, Y.; Xie, T. X.; Han, K. L.; Zhang, J. Z. H. J. Chem. Phys. 2003, 119, 12921.

31. Zhao, G. J.; Han, K. L. Chem Phys Chem 2008, 9, 1842.

32. Xie, T. X.; Zhang, Y.; Zhao, M. Y.; Han, K. L. Phys. Chem. Chem. Phys. 2003, 5, 2034.

33. Zhao, G. J.; Liu, Y. H.; Han, K. L.; Dou, Y. Chem. Phys. Lett. 2008, 453, 29.

34. Soep, B., Vetter, R. J. Phys. Chem. 1995, 99, 13569.

35. Loesch, H. J. Phys. Chem. 1997, 101, 7461.

36. Brouard, M.; Duxon, S. P.; Simons, J. P. Isr. J. Chem. 1994, 34, 67.

37. Kim, H. L.; Wickramaaratchi, M. A.; Zheng, X.; Hall, J. E. J. Chem. Phys. 1994, 101, 2033.

38. Li, Y. M. Mol. Phys. 2009, 107, 1331.

39. Chen, T. Y.; Zhang, W. P.; Wang, X. Q.; Zhao, G. J. Chem. Phys. 
2009, 365, 158.

40. Liu, Y. F.; Gao, Y. L.; Shi, D. H.; Sun, J. F. Chem. Phys. 2009, $364,46$.

41. Zhao, G. J.; Han, K. L. J. Phys. Chem. A 2007, 111, 2469.

42. Ju, L. P.; Han, K. L.; Zhang, J. Z. H. J. Comput. Chem. 2009, 30, 305.

43. Han, K. L.; Zheng, X. L.; Sun, B. F.; He, G. Z. Chem. Phys. Lett. 1991, 181, 474 .
44. Muckerman, J. T. J. Chem. Phys. 1971, 54, 1155.

45. Miranda, M. P. D.; Clary, D. C.; Castillo, J. F.; Manolopoulos, D. E. J. Chem. Phys. 1998, 108, 3142.

46. Brouard, M.; Lambert, H. M.; Rayner, S. P.; Simons, J. P. Mol. Phys. 1996, 89, 403.

47. Honvault, P.; Launay, J. M. J. Chem. Phys. 2001, 114, 1057.

48. Alexander, A. J.; Aoiz, F. J.; Bañares, L.; Brouard, M.; Short, J.; Simons, J. P. J. Phys. Chem. 1997, 101, 7544. 